\title{
Acoustic emission study of the kinetics of kink bands in the LPSO structure
}

\author{
A. Yu. Vinogradov ${ }^{1,2}$, E. V. Vasilev ${ }^{3}$, A. I. Brilevsky², D. L. Merson ${ }^{\dagger, 2}$, K. K. Kudasheva ${ }^{2}$ \\ †d.merson@tltsu.ru
${ }^{1}$ Department of Mechanical and Industrial Engineering, Norwegian University of Science and Technology, NTNU, Trondheim, N-7491, Norway
${ }^{2}$ Institute of Advanced Technologies, Togliatti State University, 14 Belorusskay St., Togliatti, 445020, Russia
${ }^{3}$ University of New Hampshire, Durham, NH, 03824, USA

\begin{abstract}
Modern magnesium alloys with the so-called long-period stacking ordered (LPSO) structure possess not only a unique microstructure, but also an excellent set of functional properties (strength, ductility, fatigue and corrosion resistance, thermal stability) that are directly or indirectly associated with the effect of the LPSO phase. In particular, the features of the nanocomposite ordering of the LPSO structure govern virtually all aspects of the mechanical behaviour of advanced alloys with such a particular structure. A characteristic feature of their plastic deformation is widely known as the formation of deformation kink bands, the behaviour of which both individually and in the interaction with each other and with lattice dislocations is not well understood. In the present work, it is proposed to use the method of acoustic emission (AE) to study these processes in real-time. The AE method revealed the presence of three simultaneously existing accommodation deformation mechanisms in model Mg-Y-Zn directly solidified crystals with LPSO structure - namely, basic dislocation slip, nucleation and growth of fault bands. Each of these processes generates a specific AE, the features of which are revealed using the original method of statistical cluster analysis of events by their power spectral density function. The growth of deformation kink bands, in contrast to the growth of twins, appears as a large-scale process of defect motion, accompanied by a reorientation of the crystal with the corresponding specific AE and activation of new channels for the basal dislocation slip, which, in the end, enables high plastic properties of the modern alloys with the LPSO structure.
\end{abstract}

Keywords: magnesium alloys, microstructure, mechanical properties, kink-band, acoustic emission.

\section{Introduction}

Nowadays, there is an increased interest in magnesium alloys containing long-period stacking ordered (LPSO) structure. These alloys possess a unique set of functional properties: a combination of extremely high specific mechanical strength, ductility, corrosion resistance and thermal stability [1-4]. The LPSO phase consists of periodic stacking of closely packed planes of (0001) hcp and $\{111\}$ fcc lattices. The important feature of the LPSO structure is that besides the topological order, there is a specific chemical order of $\mathrm{Y}$ and $\mathrm{Zn}$ atom stacking in the LPSO phase, consisting in periodic enrichment with $\mathrm{Y}$ and $\mathrm{Zn}$ atoms in certain closely packed planes [1].

A unique combination of extremely high specific strength and notable ductility (c. f. $610 \mathrm{MPa}$ and $~ 5 \%$, respectively, [2]) frequently reported for the LPSO Mg-Y-Zn alloy produced by rapid quenching is often associated with the specific strain hardening process resulting from the active occurrence and evolution of deformation kink bands $[5,6]$. Plastic strains imposed into HCP crystals in hard-slip orientations are often accommodated by mechanical twins. However, with the increase in the lattice constants ratio $(c / a)$ departing from the ideal 1.633 value (c.f. pure $\mathrm{Mg}$ has $c / a=1.624$, which is close to ideal), twinning is hindered, and plastic deformation is mainly accommodated through deformation kink bands. As has been demonstrated for Mg/LPSO alloys, no twinning is observed in the LPSO phase indeed due to specific ordering, whereas both basal slip and deformation bands, intimately interacting with each other, play a very active role in the deformation process [7]. The kink bands rotate the lattice locally by several degrees paving thereby the new ways to the enhanced dislocation slip.

Understanding the significant role that kinking processes play in the mechanical behaviour of advanced LPSO alloys stimulated intensive research of the microstructure and morphology of the kink bands. A large number of investigations have been carried out, of which those by K. Hagihara and co-authors are particularly noteworthy, c.f. [6-10]. However, the studies of the kinetics and the formation mechanisms of kink bands, and their influence on the deformation response and overall mechanical properties of magnesium alloys are still scarce, and the microscopic modelling of the kink behaviour and the associated strain hardening/softening process is an open problem [11].

There are very few in-situ methods to trace the kinetics of kinking process in a real time scale. Similar to twin formation in crystals, the avalanche-like kinking process, 
regardless of the microscopic details of the mechanism, is very fast, and involves a large volume of the material in the local rotation deformation modes. Similar to twins, the kink bands tend to extend from one grain boundary to another, and, therefore, the macroscopic kinks can reach millimetres in size. The results of direct high-speed video imaging [12] and molecular dynamics simulations [13] demonstrated that the velocity of deformation kink bands was comparable to that of twinning. Such dynamic processes are ideally suited for investigations by the acoustic emission (AE) technique, which fundamentally refers to the processes of rapid dynamic stress relaxation in local volumes of the material.

The AE method has proved to be very effective and informative in the characterisation of twinning and twinningrelated processes, e.g. $[14,15]$. Therefore, using this method to elucidate the kink evolution processes appears to be interesting and promising. An attempt is made in this paper to record and analyse the AE signal in a deforming model $\mathrm{Mg} / \mathrm{LPSO}$ crystal in order to clarify (i) the potential of the method for recognising deformation mechanisms arising in the course of deformation of the LPSO phase, (ii) their inter-relationship, and (iii) kinetics. The qualitative picture of the deformation kink band occurrence in this alloy was investigated in above-cited papers published by the Japanese researchers.

Since the AE method is indirect and integral by nature, i.e. it acquires the $\mathrm{AE}$ waveforms generated by the entire object under investigation, for reliable interpretation of $\mathrm{AE}$ information it is convenient to pair it with supporting direct imaging techniques.

\section{Experimental Procedure}

For the present first study, Mg85Y9Zn6 multi-crystals with a structure close to $100 \%$ LPSO phase were grown by directional solidification in the Bridgman furnace at $10 \mathrm{~mm} / \mathrm{h}$ rate in an Ar atmosphere, as described in detail in [6] and [16].

The original in-situ experimental setup based on the rigid Kammrath\&Weiss (Germany) screw-driven machine was used for the investigations. The setup described in $[17,18]$ allows for mechanical testing in tension, compression or bending at various temperatures with simultaneous highspeed video recording and acoustic emission measurements.

The specimens with dimensions of $4 \times 4 \times 5 \mathrm{~mm}$ were cut by the electric discharge machine along the crystallization direction so that basal slip planes of the LPSO phase were nearly parallel to the loading axis. Specimens, whose surfaces were mechanically polished to a mirror-like finish were loaded in compression at a constant cross-head speed of $10 \mu \mathrm{m} / \mathrm{s}$.

The surface microscopic images were continuously recorded using a Photron-SA3 video camera with a resolution of 1 Mpixel and a shutter speed of 33 frames per second. Since it was impossible to have an entire surface of the specimen in the field of view with the selected magnification, the central part of the specimen was observed.

The AE signals were recorded using a miniature broadband AE sensor PICO manufactured by Physical Acoustic Corporation (PAC). The sensor was securely mounted on the loading ceramic plate 3-5 mm apart from the specimen. The signal was amplified by $60 \mathrm{~dB}$ using a low-noise 2/4/6 PAC broadband amplifier with a bandwidth of $30-1200 \mathrm{kHz}$. Then it was digitised in a continuous non-threshold mode using a 16-bit PCI-2 ADC with a frequency of $2 \mathrm{MHz}$. The acquired AE stream was processed using the original algorithm of statistical, spectral and cluster analysis ASK (adaptive sequential $k$-means) proposed in [19].

\section{Results and Discussion}

Fig. 1 shows the typical microscopic images of the surface of the original as-grown multi-crystal with the LPSO structure. The grain boundaries on the top surface are well visible after etching, and the plate- or rod-shaped grains themselves are elongated in the vertical direction corresponding to the crystal growth direction. As has been clearly demonstrated by means of the of X-ray diffraction methods and transmission electron microscopy in a series of papers, see [6] and [20] for example, the grains in similar Mg-Y-Zn crystals tend to align themselves along the $\langle 11 \overline{2} 0\rangle$ crystallographic direction. Thus, the loading axis direction and the grain boundaries elongated in the longitudinal section (Fig. 1a) are nearly parallel to the basal plane (0001), which makes basal slip extremely difficult (at an ideal axial orientation, the Schmid factor is zero), and requires rapid activation of alternative deformation modes.

Fig. 2 a shows the deformation curve synchronised with the acoustic emission energy calculated from the spectral density function, as described in detail in [21]. In the specimens studied, just like that in the vast majority of magnesium alloys, AE starts at low stresses and increases sharply as the load increases. The AE signals become consistently discernible from background electric noise at normal stresses in the range of $15-20 \mathrm{MPa}$, which is lower than the critical resolved shear stresses for deformation kink band. AE becomes particularly active when a characteristic yield point is formed on the loading diagram. This kind of yield point phenomenon is systematically observed during the profuse formation of kinks. Beyond this point, the strain hardening stage is observed, at which the kinking and basal slip processes synergistically coexist supporting and promoting each other: the kink banding process itself should not be considered separately from the basal slip processes.

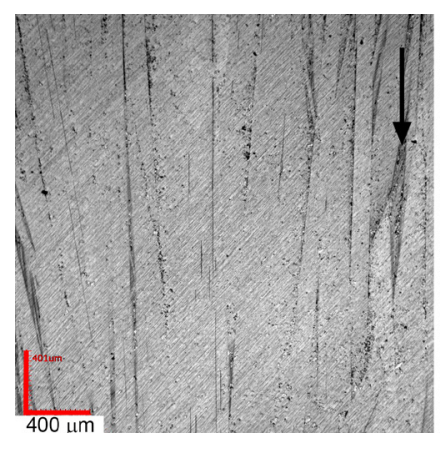

a

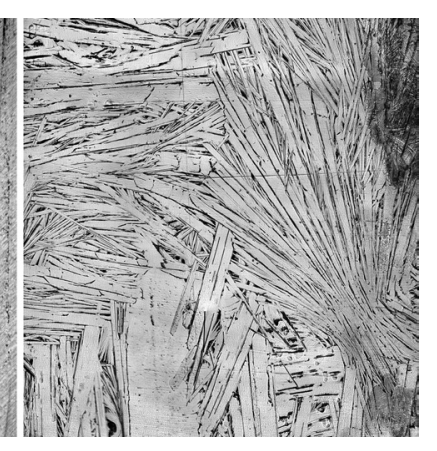

b
Fig. 1. Optical microscopic images of the longitudinal surface of the sample with the LPSO structure: the side surface from which video imaging is performed (a), the top surface (b). The arrow indicates the direction of crystal growth and the loading axis. 
In fact, dislocations in the basal plane play a key role in the occurrence of the deformation bands and their boundaries $[9,22]$.

The ridge-shaped kink bands captured in the field of view at the strain hardening stage (admittedly, not all kinks were captured, since usually the first deformation bands are observed near the stress risers close to the contact plane between the specimen and the loading plate) are clearly visible in the micrographs presented, Fig. 3, selected from the video sequence recorded during the experiment and synchronised with the loading diagram.

The kinks form almost perpendicular to the load direction. It would be more relevant to say that they form along the basal plane, which emphasises the need for the collective dislocation processes to be involved in their formation. The crystal orientation in the bands is rotated by several degrees, which is confirmed by the bending of basal slip traces. As the bands develop, convex areas appear on the specimen surface, which results in a strong black contrast obstructing the examination at relatively low magnifications, as seen in Fig. 3.

The AE energy (or power) integrally characterises the scale of underlying deformation processes. Taken alone, it, however, does not allow distinguishing between contributions from different sources (or mechanisms) which might be possibly involved in the hardening process. To enable this desired distinction, a robust algorithm termed the adaptive sequential $k$-means (ASK) was developed for categorising the signals according to the statistical similarity/dissimilarity of their power spectral density (PSD) functions [19].
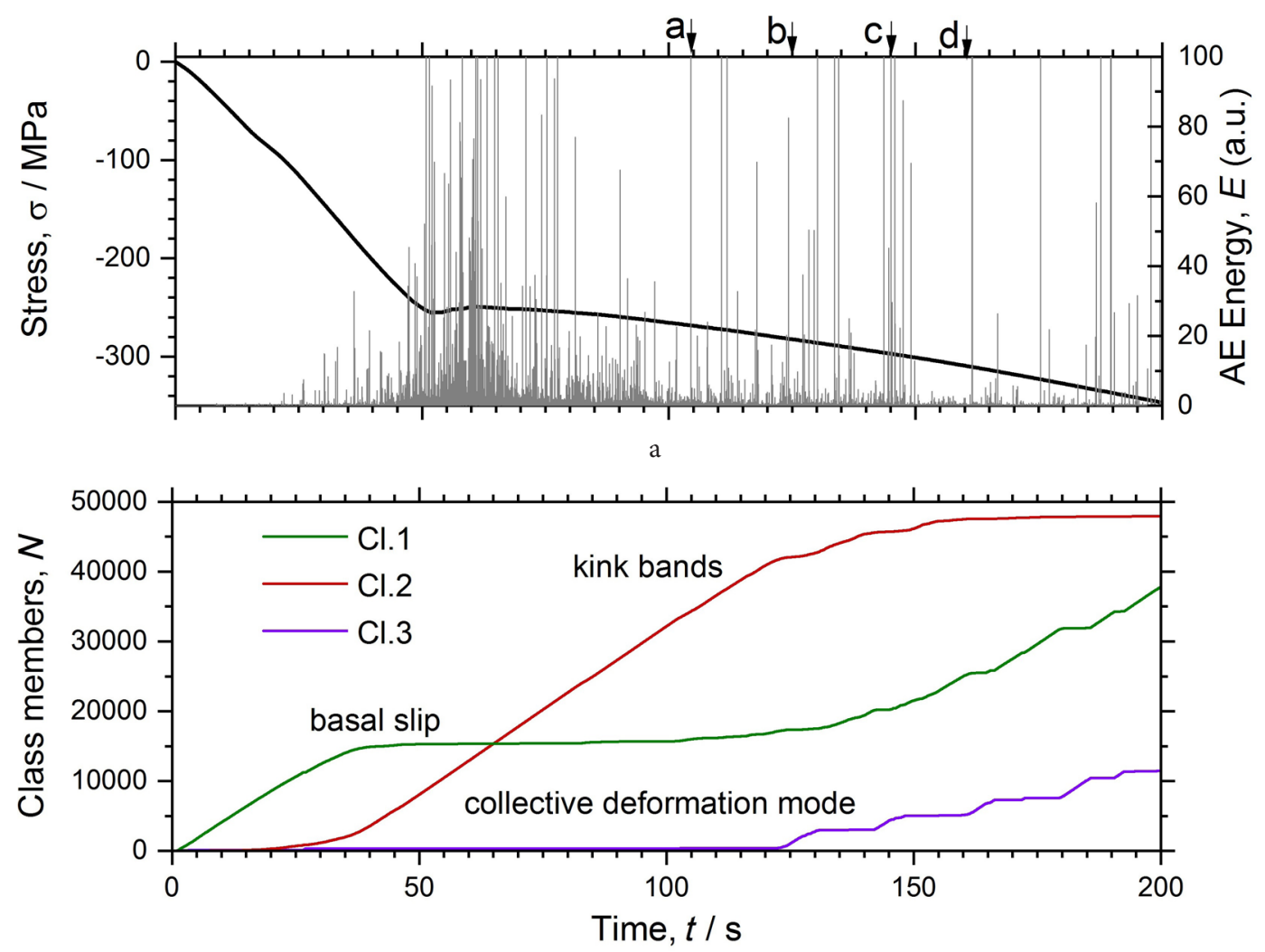

b

Fig. 2. (Color online) The loading diagram and AE energy (a) and kinetics of signal accumulation in various clusters determined by the ASK procedure (b). The background noise cluster is filtered and not shown.

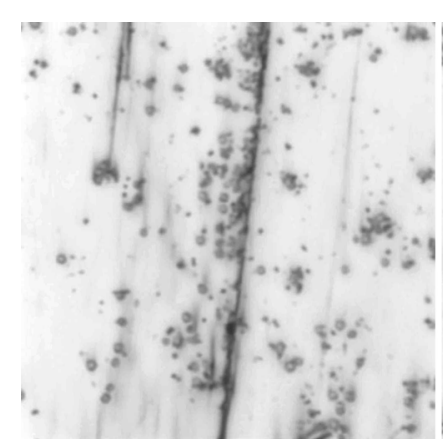

a

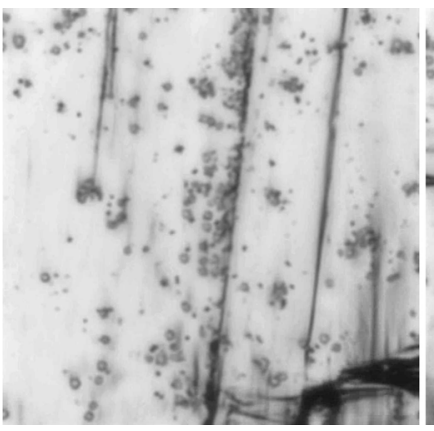

b

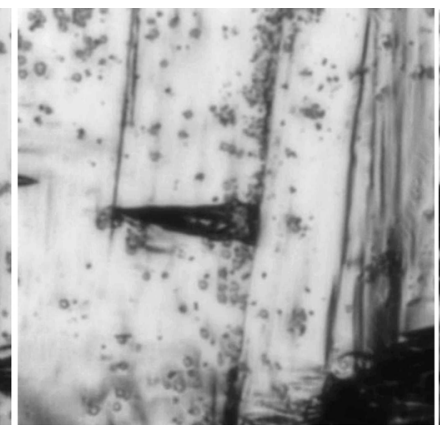

$\mathrm{C}$

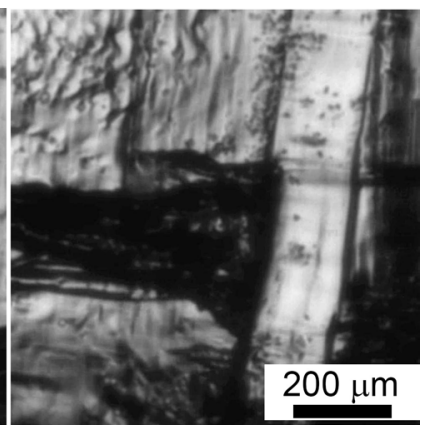

$\mathrm{d}$

Fig. 3. In-situ video images illustrating the appearance and morphology of kink bands in the LPSO crystal under study. (a) - (d) correspond to the lettering in Fig. 2 and the arrows indicating the moments of shooting. 
In brief, the method is data-driven, i.e. the number of clusters does not need to be necessarily determined $a$-priory by a supervisor. Rather, the algorithm finds the number of statistically different, maximally compact clusters from the data structure. By applying this procedure to the time series shown in Fig. 2 a, four major clusters are consistently distinguished. The first one is always the background noise, which, on the one hand, is taken as a reference point, and, on the other, is effectively filtered out by this procedure. Fig. 4 shows the normalised to unite area PSDs corresponding to the centroids of the obtained clusters. The physically meaningful clusters 1-3 (Fig. 4b-d, respectively) are evidently different from the noise (Fig. $4 \mathrm{a}$ ) and are different among each other in their AE spectral composition.

Once the signal categorisation has been performed and every fragment of the stream is assigned to a dominant deformation mechanism, it is easy to trace the kinetics of their evolution independently. Fig. $2 \mathrm{~b}$ shows the event accumulation curves for individual clusters. A complex interplay between the occurring processes is revealed. The $\mathrm{AE}$ process commences at low stresses, and Cluster 1 comprising of relatively low amplitude events shows up. Since there are no deformation kink bands at such stresses, and since not all grains are perfectly parallel to the basal planes, basal slip can occur locally (see, e.g. $[9,23]$ ). Therefore, Cluster 1 can be logically associated with the local basal slip. The process of signal accumulation in Cluster 1 rapidly grows and reaches a plateau. However, this does not mean that the dislocation slip process has ceased. The AE cluster analysis suggests that multiple mechanisms can operate concurrently during strain hardening. While the constrained dislocation slip evolves slowly, the new mechanism comes into play at approximately $25^{\text {th }}$ second and is termed Cluster 2 by order of appearance. Its activity and the AE energy are by far higher than those of dislocation slip (Cluster 1). Therefore, its contribution to the overall AE signal prevails over Cluster 1. Once activated, the mechanism corresponding to Cluster 2 progresses until the

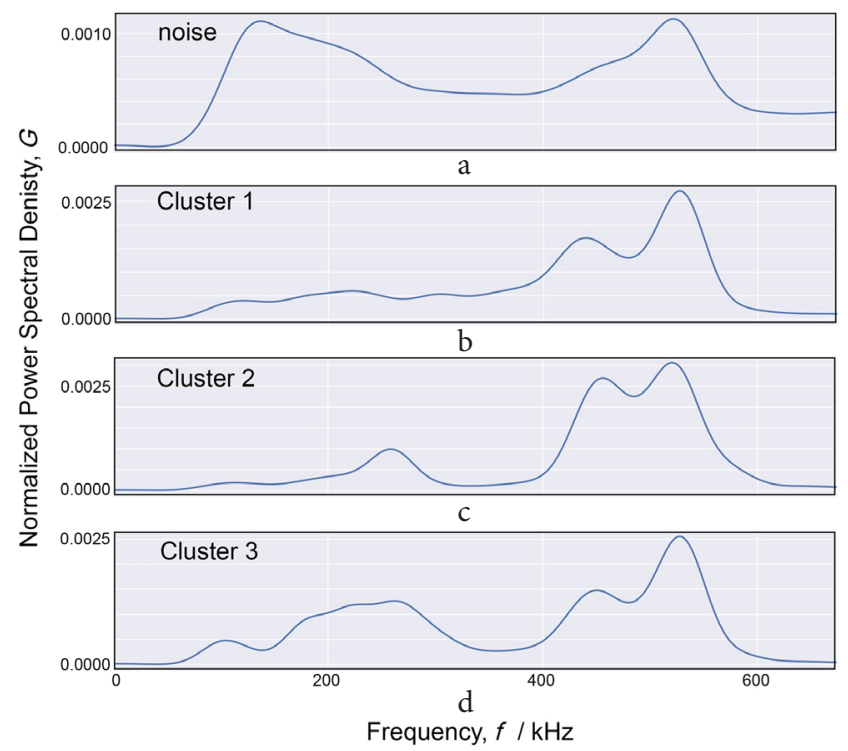

Fig. 4. Centroids of clusters of AE events noise (a) and deformation processes (b-d) determined by the degree of statistical similarity of spectral density functions by the ASK method. end of loading. The high activity and energy of this process, which peaks at the characteristic yield point on the loading diagram, suggests that the powerful AE corresponding to Cluster 2 is generated due to the formation and growth of kink bands, which are then appeared in the field of view of the video imaging device, Fig. 3. The occurrence of Cluster 3 signals looks most intriguing and unusual at the stage of mature plastic deformation when very large kinks form and dominate the overall strain hardening response. The step-wise kinetics of event accumulation in this cluster resembling in a sense the behaviour of Portevin-Le Chatelier bands is very specific. Comparison of the video sequence and the kinetics of this cluster signal occurrence allows one to state with high confidence that the occurrence of Cluster 3 is associated with the development of large deformation kink bands. Unlike the microscopic but rapidly initiating bands, the "growth" of large kinks is relatively slow. However, since such largescale events involve a huge number of dislocations and/or possibly disclinations as natural crystal defects describing rotational deformation modes including kinks [24], it is not surprising that AE (even though of relatively small amplitude and energy) is generated, reflecting the evolution process of such large deformation elements. The occurrence and development of coarse kinks re-orientates the lattice and, consequently, facilitates the dislocation slip, which entails the "reactivation" of Cluster 1 signals whose accumulation kinetics occurs almost in-phase with the Cluster 3 signals, Fig. 2 b. Let us notice that Cluster 3 is significantly lower in the frequency content compared to Cluster 1, Fig. 4, which emphasises the higher scale level of its sources.

Thus, this brief communication, in our view, exposes a new in-situ approach to the investigation of kink band-related processes. Based on these preliminary results, in the nearest future, we shall present elsewhere a more elaborated analysis and interpretation of the kink band evolution.

\section{Conclusions}

In the course of plastic deformation of magnesium alloys with the distinct LPSO structure, the dislocation slip and kink formation processes coexist, exhibiting a complex relationship with each other. The modern AE method makes it possible to discriminate between these processes reliably in a real time scale. The obtained results support the hypothesis that even under conditions of hindered dislocation slip, the collective dislocation slip processes play an essential role in the initiation and propagation of deformation kink bands. Evolution of kink bands, unlike conventional twin thickening in magnesium and its alloys, is a large-scale process involving massive defect motion with a necessary local crystal re-orientation, which is accompanied by the specific AE reflecting many fine details of the temporal kink development. Local rotational reorientation of the crystal lattice in the kink bands creates new channels for basal dislocation slip, which eventually results in good ductility of $\mathrm{Mg} / \mathrm{LPSO}$ alloys.

Acknowledgements. The financial support is provided by the Ministry of Education and Science of the Russian Federation within the state assignment 3.3881.2017/4.6. The authors are 
grateful to prof. Y. Kawamura (Magnesium Research Center, Kumamoto University) for providing materials for this study and for his stimulating interest in this work.

\section{References}

1. E. Abe, Y. Kawamura, K. Hayashi, A. Inoue. Acta Materialia. 50 (15), 3845 (2002). Crossref

2. Y. Kawamura, K. Hayashi, A. Inoue, T. Masumoto. Materials Transactions. 42 (7), 1172 (2001). Crossref

3. Y. Kawamura, T. Kasahara, S. Izumi, M. Yamasaki. Scripta Materialia. 55 (5), 453 (2006). Crossref

4. M. Yamasaki, S. Izumi, Y. Kawamura, H. Habazaki. Applied Surface Science. 257 (19), 8258 (2011) . Crossref

5. X.H. Shao, Z. Q. Yang, X. L. Ma. Acta Materialia. 58 (14), 4760 (2010). Crossref

6. K. Hagihara, N. Yokotani, Y. Umakoshi. Intermetallics. 18 (2), 267 (2010) . Crossref

7. K. Hagihara, A. Kinoshita, Y. Fukusumi, M. Yamasaki, Y. Kawamura. Materials Science and Engineering: A. 560, 71 (2013). Crossref

8. K. Hagihara, A. Kinoshita, Y. Sugino, M. Yamasaki, Y. Kawamura, H. Y. Yasuda, Y. Umakoshi. Acta Materialia. 58 (19), 6282 (2010). Crossref

9. K. Hagihara, A. Kinoshita, Y. Sugino, M. Yamasaki, Y. Kawamura, H. Y. Yasuda, Y. Umakoshi. Intermetallics. 18 (5), 1079 (2010). Crossref

10. K. Hagihara, T. Okamoto, H. Izuno, M. Yamasaki, M. Matsushita, T. Nakano, Y. Kawamura. Acta Materialia. 109, 90 (2016). Crossref

11. K. Hagihara, M. Yamasaki, M. Honnami, H. Izuno, M. Tane, T. Nakano, Y. Kawamura. Philosophical Magazine. 95 (2), 132 (2015). $\underline{\text { Crossref }}$
12. Y. Muto, T. Shiraiwa, M. Enoki. Materials Science and Engineering: A. 689, 157 (2017). Crossref

13. R. Matsumoto, M. Uranagase, N. Miyazaki. Materials Transactions. 54 (5), 686 (2013). Crossref

14. A. Vinogradov, D. Orlov, A. Danyuk, Y. Estrin. Acta Materialia. 61 (6), 2044 (2013). Crossref

15. A. Vinogradov, K. Máthis. JOM. 68 (12), 3057 (2016). Crossref

16. M. Tane, Y. Nagai, H. Kimizuka, K. Hagihara, Y. Kawamura. Acta Materialia. 61 (17), 6338 (2013). $\underline{\text { Crossref }}$

17. M. Seleznev, A. Vinogradov. Review of Scientific Instruments. 85 (7), 076103 (2014). $\underline{\text { Crossref }}$

18. A. Vinogradov, E. Vasilev, M. Seleznev, K. Máthis, D. Orlov, D. Merson. Materials Letters. 183, 417 (2016). Crossref

19. E. Pomponi, A. Vinogradov. Mech. Syst. Signal Proc. 40 (2), 791 (2013). Crossref

20. S. Yoshimoto, M. Yamasaki, Y. Kawamura. Materials Transactions. 47 (4), 959 (2006). Crossref

21. A. Vinogradov, M. Nadtochiy, S. Hashimoto, S. Miura. Materials Transactions JIM. 36 (4), 496 (1995). Crossref

22. T. Matsumoto, M. Yamasaki, K. Hagihara, Y. Kawamura. Acta Materialia. 151, 112 (2018). Crossref

23. K. Hagihara, A. Kinoshita, Y. Sugino, M. Yamasaki, Y. Kawamura, H. Y. Yasuda, Y. Umakoshi. Transactions of Nonferrous Metals Society of China. 20 (7), 1259 (2010). Crossref

24. M.Y. Gutkin, K.N. Mikaelyan, A.E. Romanov, P. Klimanek. Physica Status Solidi (A) Applied Research. 193 (1), 35 (2002). ․ㅏossref

25. E. Abe, Y. Kawamura, K. Hayashi, A. Inoue. Acta Materialia. 50 (15), 3845 (2002). $\underline{\text { Crossref }}$ 